\title{
Assessing the diet of adolescent girls in the UK
}

\author{
Siân Robinson*, Ria Skelton, Mary Barker and Clare Wilman \\ MRC Environmental Epidemiology Unit, University of Southampton, Southampton SO 16 6YD, UK
}

Submitted 20 January 1999: Accepted 7 June 1999

\begin{abstract}
Objective: To assess the ability of a food frequency questionnaire (FFQ1) and a food checklist (FCL) to determine energy and macronutrient intakes of adolescent girls in the general population.

Design: Energy and macronutrient intakes determined by FFQ1 and the FCL were compared with those from a 7-day weighed dietary record (WDR). The reproducibility of FFQ1 was assessed by a comparison of intakes with those from a repeated questionnaire (FFQ2) completed a month later.

Setting: Southampton, UK.

Subjects: Forty-seven 15-year-old girls completed FFQ1 and the WDR and FCL; and 61 girls completed FFQ1 and FFQ2.

Results: The broad dietary patterns described by the three methods of assessment were similar, although absolute intakes differed. Energy and macronutrient intakes determined by FFQ1 were higher than those recorded in the WDR (all $P<0.001$ ), but intakes assessed by the FCL and WDR were similar. Only FFQ1-assessed energy intakes appeared consistent with predicted energy requirements. With the exception of protein intake, there was reasonable agreement between FFQ1 and the WDR in their estimation of energy and macronutrient intake (range of correlation coefficients 0.28 for energy to 0.33 for carbohydrate). The poorer agreement between FFQ1 and the WDR in their estimates of protein intake arose principally from the misclassification of meat and fish intake, although there was no obvious explanation for this. Energy and macronutrient intakes were similar for FFQ1 and FFQ2.

Conclusions: Adolescent girls give reproducible answers in response to a selfadministered FFQ, which yield useful information about their broad dietary patterns. The FFQ may be a more suitable dietary assessment method than prospective records for use in general population studies of girls of this age. We discuss suggestions for its improved performance.
\end{abstract}

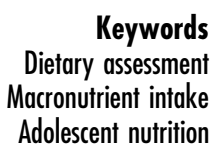

Despite the interest in the relationship between diet and disease, little is known about how and when dietary patterns become established and what influences their development. If eating habits formed early in life remain with an individual, it is important that we understand more about how dietary patterns evolve. In a recent study we found that specific patterns of macronutrient intake in pregnancy were associated with differences in fetal and placental weight at birth ${ }^{1}$. Since we wanted to find out whether these dietary patterns were also evident in much younger (adolescent) women in the general population, we needed a method of dietary assessment suitable for use by this age group.

The use of weighed prospective recording techniques by adolescents has been shown to be associated with the underreporting of intake when compared with estimates of total energy expenditure ${ }^{2,3}$. Although the diet history method has been used successfully to record adolescent diet $^{4}$, individual interviews are needed which may limit the number of individuals included in the study. More recently, FFQs have been developed for the assessment of adolescent diet in Norway ${ }^{5}$ and in the United States ${ }^{6}$, and in this paper we report on the use of a FFQ by adolescents in the UK.

In addition to the FFQ that we designed (FFQ1), we developed a FCL for the study. FFQ1 assessed diet retrospectively over a month, whilst the FCL (which included the same list of foods as the questionnaire) was used to record food intake prospectively over a week. Our objective was to develop a method of dietary assessment which describes the dietary pattern of adolescent girls in the general population, which can be used to estimate energy and macronutrient intake, and which is widely accepted and suitable for use in large-scale studies.

In this paper we evaluate FFQ1 and the FCL by comparing food, energy and macronutrient intakes assessed by both methods with those determined using a 7-day WDR. We also examine the reproducibility of 
the FFQ by comparison of FFQ1-determined intakes with those from a second questionnaire (FFQ2) completed 1 month later.

\section{Subjects and methods}

\section{Subjects and study design}

Between October 1995 and July 1996, girls in years 10 and 11 at schools in Southampton (UK) were invited to take part in the study. The girls were seen in small groups by the same investigator (RS). The FFQ1 was completed at the first visit and, although FFQ1 was selfadministered, the investigator was present during its completion and help was given if needed. Following this visit the girls were asked to record their food/ drink intake prospectively over 7 days using either the WDR or the FCL. The order of completion of these prospective records was alternated for each group. At the end of the first recording period a second visit took place for collection of the records, and for instruction in the completion of the next 7-day record. At a third visit the second FFQ (FFQ2) was distributed. The girls were asked to complete this a month after the initial visit and to return it by post. Throughout the dietary recording periods the girls were encouraged to contact the investigator for any support or help they needed.

At the second visit additional information was collected relating to the girl's age and background. Social class was defined according to the occupation of the girl's head of household ${ }^{7}$. Basal metabolic rate (BMR) was estimated using a standard equation ${ }^{8}$ from the girl's weight which was measured at the third visit (Seca scale).

Ethical approval for the study was given by the Southampton and South West Hampshire Health Authority Joint Ethics Committee, and both parents and girls gave their informed consent.

\section{Food frequency questionnaire}

A list of 83 foods or food groups was compiled which would contribute to at least $90 \%$ of the intake of energy and macronutrients of adolescent girls in our population. The list was based on dietary data from food diaries kept by 600 pregnant women in the same population ${ }^{9}$, and was revised in light of focus group discussion with adolescent girls. Portion sizes were obtained from the above dataset or from published lists $^{10,11}$. The questionnaire was self-administered, and each girl was asked to estimate her average frequency of consumption of each food/group over the previous month by selecting one of nine frequencies ranging from never to more than 5 times a day. At the end of the questionnaire the girl was asked to record any additional foods which had been consumed once a week or more which had not been included in the list of 83 foods.

\section{Food checklist}

The same list of foods as the FFQ was used in the FCL. Each girl was given a booklet containing seven separate lists, one for each day of recording. The girl was asked to 'tick' the food on every occasion she consumed it throughout the day, and to record the total number of occasions at the end of each day. At the end of each day's list there was a space for food/drinks which had been consumed but which were not represented on the food list. The FCL was completed on 7 consecutive days.

\section{Weighed dietary record}

Each girl was given a set of dietary scales (Soehnle) and an open diary in which to record all food/drinks consumed. She was instructed in the use of the scales and was encouraged to weigh as much of her food/ drinks as possible during the recording period. When this was not possible, food weights were estimated using standard portion sizes ${ }^{10,11}$; or for foods from the school canteen, portion size information was obtained from the catering staff. The WDR was completed on 7 consecutive days.

\section{Data analysis}

Eighty girls volunteered to take part in the study and all completed FFQ1. Seventy girls (88\%) returned a completed FCL, $48(60 \%)$ returned a complete 7-day food diary and $61(76 \%)$ returned a completed FFQ2.

Nutrient intakes were calculated by multiplying the portion weight (FFQ1, FFQ2, FCL) or the weight of food recorded (WDR) by its nutrient content ${ }^{12-15}$. Any additional foods recorded in FFQ1 and FFQ2 or the FCL were treated the same way and added to intakes from the foods on the standard list. Energy and macronutrient intakes were normally distributed.

Since we have previously observed overreporting of intake using an administered $\mathrm{FFQ}^{9}$, in the present study we defined a priori that an implausible response to the FFQ was an energy intake : predicted BMR ratio greater than 3.0. Whilst the principal aim of a FFQ is to rank individuals within the distribution of nutrient intake, we interpret energy intakes above this defined level as an indication that the subject has failed to understand how to complete the FFQ and this is the reason for their exclusion.

On this basis one girl was excluded since her ratio of energy intake (from FFQ1) to predicted BMR was 3.50. We report data for 47 girls (59\%) who completed the first three parts of the study, and examine the reproducibility of FFQ1 by comparing energy and macronutrient intakes with those determined by FFQ2 for 61 girls (76\%) who completed it a month later.

The different assessment methods were compared in terms of their estimates of energy and macronutrient intakes using paired $t$-tests, by calculating Pearson 
Table 1 Characteristics of girls in the study $(n=47)$

\begin{tabular}{lrrc}
\hline & Mean & SD & Range \\
\hline Age (years) & 15.7 & 0.6 & $14.2-16.6$ \\
Weight $(\mathrm{kg})$ & 56.7 & 8.3 & $36.3-82.7$ \\
Height $(\mathrm{cm})$ & 163.4 & 6.2 & $146.2-176.6$ \\
Body mass index $\left(\mathrm{kg} \mathrm{m}^{-2}\right)$ & 21.2 & 2.6 & $15.2-29.2$ \\
\hline
\end{tabular}

correlation coefficients, and limits of agreement between methods were examined using BlandAltman plots ${ }^{16}$.

\section{Results}

Characteristics of the 47 girls are given in Table 1. Sixtyone per cent were from social classes I and II, 26\% came from social classes IIIN and M, and 9\% came from class IV. Social class could not be classified for three girls. Energy and macronutrient intakes assessed by each dietary method are shown in Table 2 . Absolute intakes determined by FFQ1 were greater than those from the WDR (all $P<0.001$ ), but with the exception of protein intake $(P=0.003)$, energy and macronutrient intakes assessed by the WDR and FCL did not differ. The energy intake to predicted BMR ratios from the WDR were lower than those estimated from FFQ1 $(P<$ 0.001 ) but the ratios for the WDR and FCL were similar. The proportion of energy intake from fat and carbohydrate did not differ when comparing either FFQ1 or the FCL with the WDR, but in each case, the proportion of energy from protein was less than that determined by the WDR (FFQ1-WDR, $P=0.008$; FCLWDR, $P=0.017$ ).

Bland-Altman plots ${ }^{16}$ were used to examine the limits of agreement between FFQ1 and the WDR and between the FCL and the WDR in their estimation of energy and macronutrient intake. For each individual, the difference in absolute intake between the two methods was plotted against their average intake from the same methods (plots for energy intake are shown in Fig. 1; macronutrients are not shown). For FFQ1 and the WDR, as the average level of energy intake rose the difference in intake between the methods increased $(P$ $<0.001)$. This was also the case for each macronutrient (all $P<0.001$ ), and suggests overreporting by FFQ1 at higher levels of intake. This pattern was not evident for any of the macronutrients when expressed as a proportion of energy intake, or for any of the comparisons between the FCL and the WDR.

The differences in macronutrient intake and the correlation coefficients calculated for the FFQ1-WDR and FCL-WDR comparisons are given in Table 3. With the exception of protein intake, there was reasonable agreement between FFQ1 and the WDR in their estimates of energy and macronutrient intake. Correlation coefficients for the FCL with the WDR were generally greater than those seen for the FFQ1 comparison with the WDR.

Although there were clear differences between methods in their assessment of energy and macronutrient intake, at the group level the broad profile of diet described by the three methods was similar (Fig. 2). When expressed as a proportion of energy intake, relatively greater intakes of soft drinks and fruit were recorded by FFQ1 whilst relatively high intakes of cereal products were recorded in the WDR.

Table 4 shows the differences in energy intake between assessment methods according to different food groups, and the correlation coefficients for those groups. Higher energy intakes from bread, fruit, dairy products and drinks were recorded by FFQ1 when compared with the WDR, but in terms of correlation, the poorest agreement between FFQ1 and the WDR was seen for cereal products, meat and fish, and snack foods (confectionery, chocolate and savoury snacks) and sugar. There was no consistent pattern in the absolute differences in intake according to food group when comparing the FCL and WDR, but in each case the correlations observed between methods were higher than those for FFQ1 in comparison with the WDR.

To investigate further why protein intakes appeared to be assessed more poorly by FFQ1 than the other

Table 2 Daily energy and macronutrient intakes according to dietary assessment method $(n=47)$

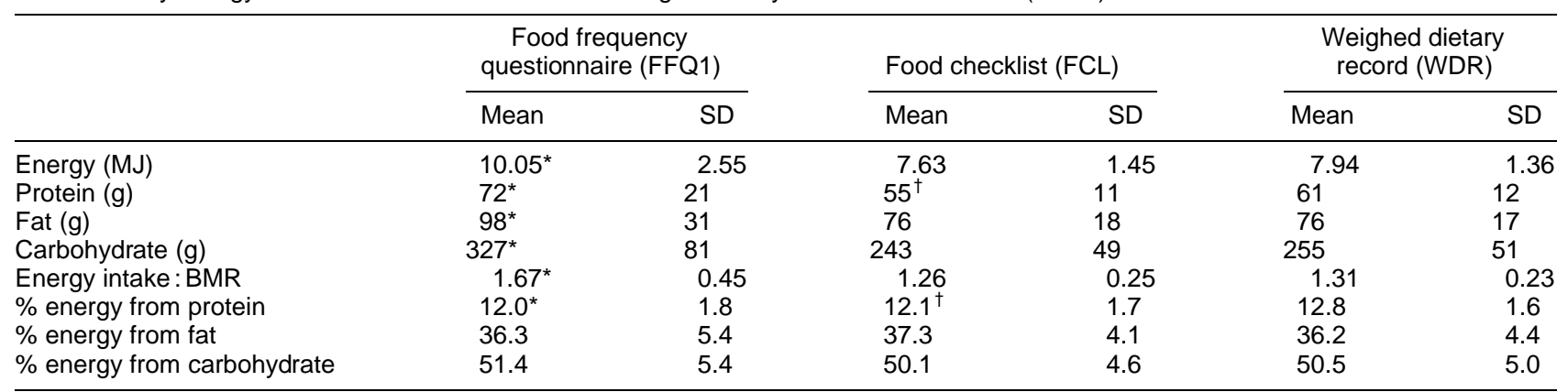

${ }^{*} P<0.01$ FFQ1 compared with WDR.

${ }^{\dagger} P<0.02$ FCL compared with WDR. 


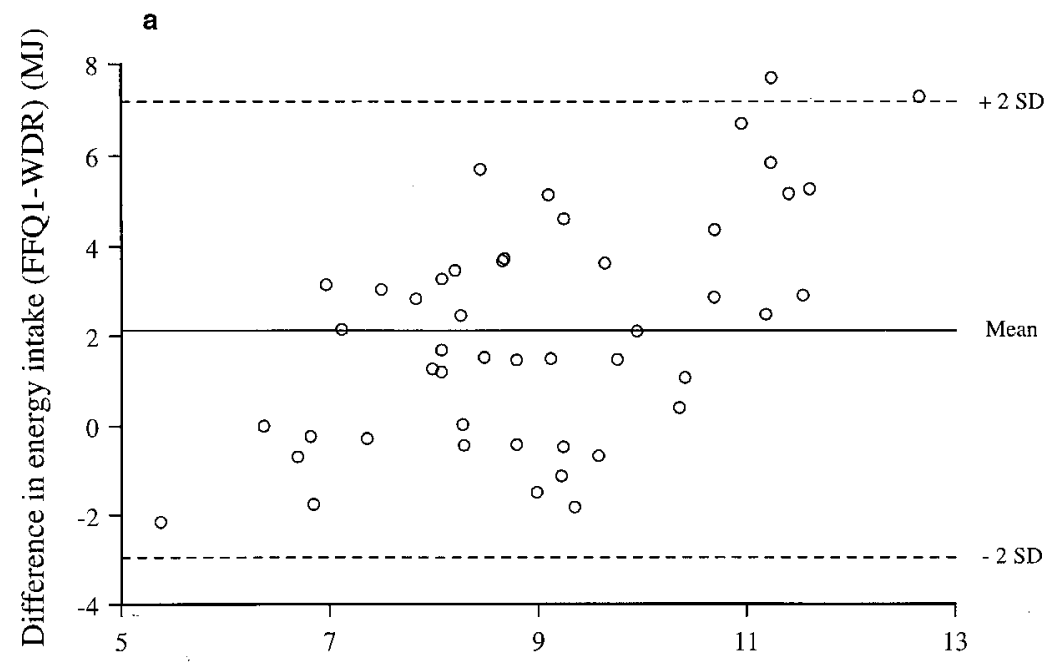

Mean energy intake (MJ)

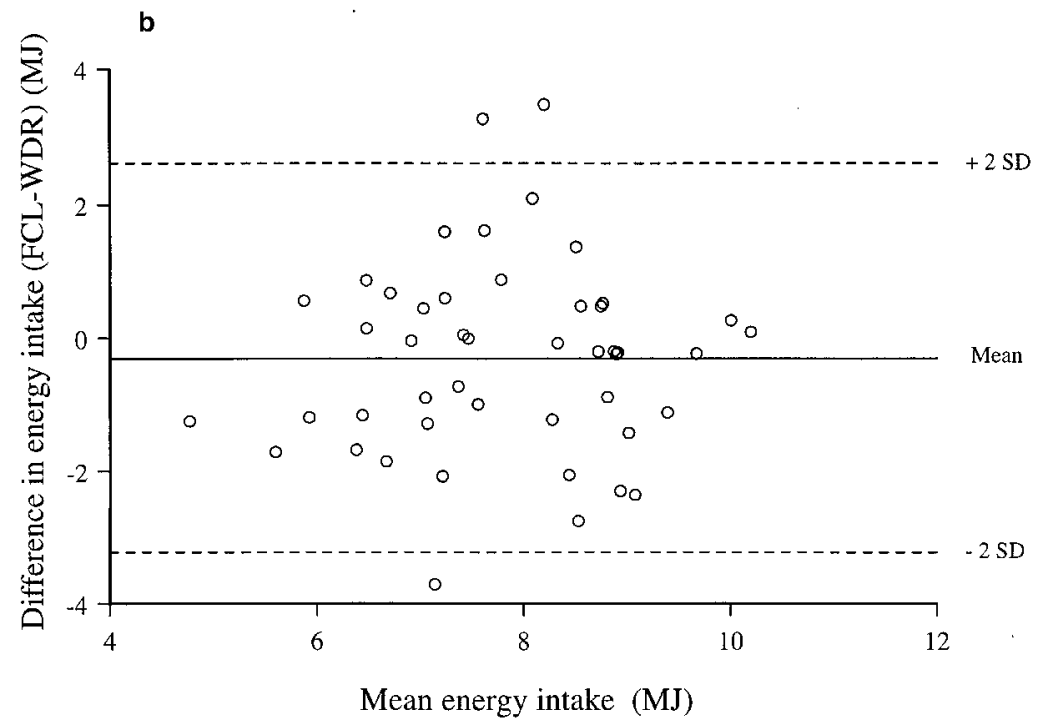

Fig. 1 Difference in energy intake between (a) FFQ1 and the WDR, and (b) the FCL and WDR plotted against average intake from the two methods

Table 3 Differences in energy and macronutrient intakes and correlation coefficients for comparisons of FFQ1 and the FCL with the WDR $(n=47)$

\begin{tabular}{|c|c|c|c|c|c|c|c|c|}
\hline & \multicolumn{4}{|c|}{ FFQ1-WDR } & \multicolumn{4}{|c|}{ FCL-WDR } \\
\hline & Difference & SD & $r$ & $P^{\mathrm{a}}$ & Difference & SD & $r$ & $P^{\mathrm{b}}$ \\
\hline Energy (MJ) & 2.12 & 2.53 & 0.28 & 0.056 & -0.31 & 1.46 & 0.46 & 0.001 \\
\hline Protein (g) & 11.5 & 22.9 & 0.13 & 0.395 & -6.1 & 13.4 & 0.33 & 0.024 \\
\hline Fat $(\mathrm{g})$ & 21.8 & 30.4 & 0.30 & 0.041 & -0.3 & 18.6 & 0.44 & 0.002 \\
\hline Carbohydrate (g) & 71.2 & 80.6 & 0.33 & 0.025 & -12.3 & 48.4 & 0.53 & 0.001 \\
\hline$\%$ energy from protein & -0.81 & 1.98 & 0.30 & 0.044 & -0.72 & 2.00 & 0.24 & 0.111 \\
\hline$\%$ energy from fat & 0.17 & 5.52 & 0.38 & 0.009 & 1.13 & 4.27 & 0.50 & 0.001 \\
\hline$\%$ energy from carbohydrate & 0.88 & 6.17 & 0.30 & 0.042 & -0.40 & 4.72 & 0.52 & 0.001 \\
\hline
\end{tabular}

a $P$ values for correlations between FFQ1 and the WDR

${ }^{\mathrm{b}} P$ values for correlations between FCL and the WDR. 


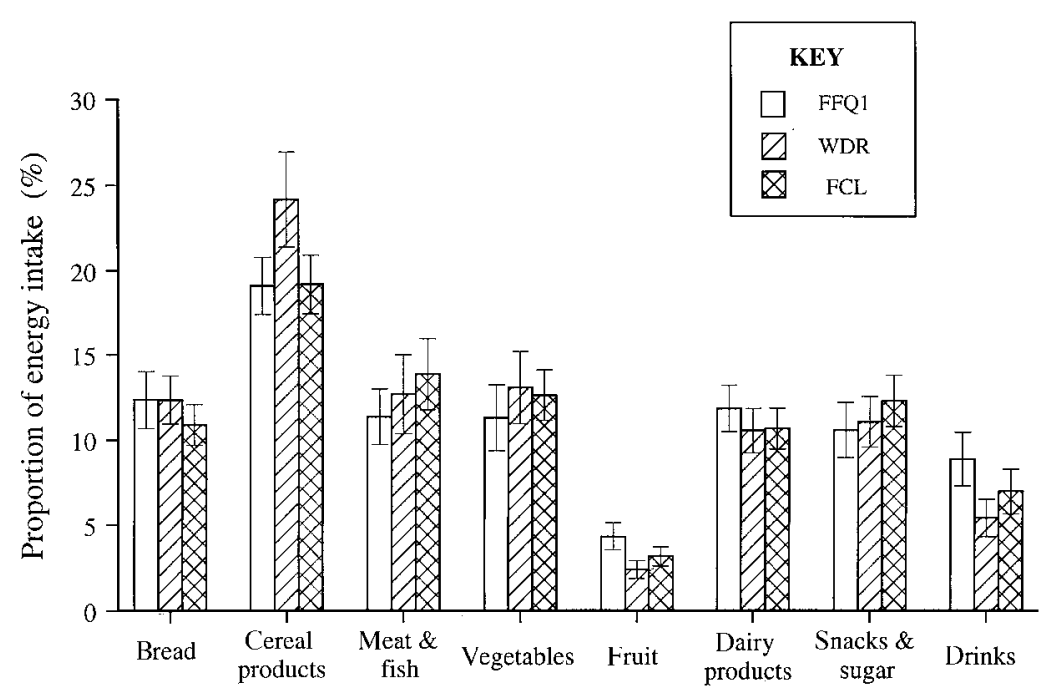

Fig. 2 The proportion of energy intake derived from the main food groups according to assessment method (mean value, 95\% $\mathrm{Cl}$ )

macronutrients, total protein intake was subdivided into meat and fish, dairy and cereal protein. Although at the group level there was good agreement between FFQ1 and the WDR in their estimation of meat and fish intake (Fig. 2), there was considerable misclassification of individuals within the distribution of meat and fish intake when FFQ1 was compared with the WDR (Table 4). Portion sizes of meat and fish were similar for FFQ1 and the WDR, and the misclassification arose principally from differences in the reported frequency of consumption. As a result, there was a lower correlation coefficient for total protein intake $(r=0.13)$ than that for non-meat and fish (cereal and dairy) protein intake ( $r=0.36, P=0.014)$. There was no obvious reason for the misclassification of individuals within the distribution of meat and fish intake.

We assessed the reproducibility of FFQ1 by comparing energy and macronutrient intakes with those determined by FFQ2 completed 1 month later. The girls $(n=61)$ who returned $\mathrm{FFQ} 2$ were similar in characteristics (mean age 15.6 years (SD 0.6); body mass index $21.1 \mathrm{~kg} \mathrm{~m}^{-2}$ (SD 2.9)) and social class distribution (62\% in classes I and II) to the smaller group of girls who completed the WDR (Table 1). Table 5 shows energy and macronutrient intake and proportion of energy from protein, fat and carbohydrate estimated by FFQ1 and FFQ2, together with correlation coefficients for their comparisons. There were no significant differences in intake of energy or any macronutrient between FFQ1 and FFQ2.

\section{Discussion}

We compiled a list of 83 foods which contribute to more than $90 \%$ of the energy and macronutrients in the diet of adolescent girls in our population. This list of foods was used in FFQ1 to assess frequency of consumption retrospectively over a month, and in a FCL to record intake prospectively each day for a week. Our aim was to develop a dietary assessment method which could be used by adolescent girls in the general population. This paper reports on the evaluation of FFQ1 and

Table 4 Differences in energy intake (MJ) and correlation coefficients according to food group for comparisons of FFQ1 and the FCL with the $\operatorname{WDR}(n=47)$

\begin{tabular}{|c|c|c|c|c|c|c|c|c|}
\hline & \multicolumn{4}{|c|}{ FFQ1-WDR } & \multicolumn{4}{|c|}{ FCL-WDR } \\
\hline & Difference & SD & $r$ & $P^{a}$ & Difference & SD & $r$ & $P^{\mathrm{b}}$ \\
\hline Bread & $0.27^{*}$ & 0.60 & 0.43 & 0.002 & $-0.14^{\dagger}$ & 0.32 & 0.62 & 0.001 \\
\hline Cereal products & -0.05 & 1.10 & 0.20 & 0.181 & $-0.49^{\dagger}$ & 0.85 & 0.43 & 0.002 \\
\hline Meat and fish & 0.13 & 0.72 & 0.26 & 0.079 & 0.06 & 0.51 & 0.62 & 0.001 \\
\hline Vegetables & 0.13 & 0.69 & 0.46 & 0.001 & -0.06 & 0.52 & 0.37 & 0.010 \\
\hline Fruit & $0.24^{*}$ & 0.31 & 0.38 & 0.009 & $0.05^{\dagger}$ & 0.12 & 0.66 & 0.000 \\
\hline Dairy products & $0.37^{*}$ & 0.58 & 0.34 & 0.018 & -0.02 & 0.43 & 0.38 & 0.008 \\
\hline Snacks and sugar & 0.18 & 0.65 & 0.23 & 0.129 & 0.08 & 0.49 & 0.43 & 0.003 \\
\hline Drinks & $0.44^{*}$ & 0.52 & 0.32 & 0.027 & $0.09^{\dagger}$ & 0.23 & 0.74 & 0.000 \\
\hline
\end{tabular}

${ }^{\text {a }} P$ values for correlations between FFQ1 and the WDR.

${ }^{\mathrm{b}} P$ values for correlations between FCL and the WDR.

${ }^{*} P<0.005$ difference between FFQ1 and the WDR.

${ }^{\dagger} P<0.01$ difference between the FCL and the WDR. 
Table 5 Daily energy and macronutrient intakes determined by FFQ1 and FFQ2 $(n=61)$

\begin{tabular}{|c|c|c|c|c|c|c|}
\hline & \multicolumn{2}{|c|}{ FFQ1 } & \multicolumn{2}{|c|}{ FFQ2 } & \multirow[b]{2}{*}{$r$} & \multirow[b]{2}{*}{$P$} \\
\hline & Mean & SD & Mean & SD & & \\
\hline Energy (MJ) & 10.50 & 2.39 & 10.15 & 3.05 & 0.45 & 0.000 \\
\hline Protein $(\mathrm{g})$ & 75 & 19 & 70 & 22 & 0.44 & 0.000 \\
\hline Fat $(g)$ & 104 & 31 & 100 & 37 & 0.52 & 0.000 \\
\hline Carbohydrate $(\mathrm{g})$ & 340 & 77 & 330 & 100 & 0.48 & 0.000 \\
\hline Energy intake:BMR & 1.72 & 0.44 & 1.66 & 0.53 & 0.51 & 0.000 \\
\hline$\%$ energy from protein & 11.9 & 1.7 & 11.6 & 1.5 & 0.67 & 0.000 \\
\hline$\%$ energy from fat & 36.8 & 5.2 & 36.9 & 5.8 & 0.78 & 0.000 \\
\hline$\%$ energy from carbohydrate & 51.1 & 5.3 & 51.3 & 6.0 & 0.76 & 0.000 \\
\hline
\end{tabular}

the FCL by comparing estimates of energy and macronutrient intake with those determined from a 7-day WDR.

Whilst a large group of girls agreed to take part in our evaluation study, only 59\% managed to complete all parts of the study, and the group is dominated by girls from higher social classes. The girls in the present study must be considered volunteers and clearly such a group cannot be representative of the general population. Given the highly motivated nature of our subjects we might expect the comparison of FFQ1 and the FCL with the WDR to represent 'best estimates' which do not inform us about the validity of the methods when used by a more heterogeneous group. Conversely the homogeneity within such a self-selected group may lead to an underestimation of the ability of FFQ1 and the FCL to describe and discriminate diets of wider variability ${ }^{17}$.

Despite the compliant nature of the girls, their energy intakes assessed by the WDR appeared low in relation to predicted BMR. In a recent review of studies in which total energy expenditure (TEE) of girls of this age has been measured using doubly-labelled water, the ratio of TEE to BMR ranges from 1.68 to $1.79^{3}$. Torun et al. ${ }^{18}$ propose a minimum value of TEE : BMR ratio of 1.30 for girls, with the suggestion that dietary records which aim to assess habitual intake should be excluded if they yield lower ratios of energy intake to BMR. In the present study, using 1.30 as a cut-off for the ratio of energy intake to predicted BMR would result in the loss of 23 (49\%) of the records.

Such low energy intakes, although comparable with other studies using prospective weighed recording techniques ${ }^{2,3,19}$, cannot represent habitual diet. Although it is possible that energy intakes were low during the study period, the number of low energy reporters in the group is suggestive of some underreporting of food intake. Livingstone et $a l^{2}{ }^{2}$ also describe underreporting of intake by adolescents in their study and attributed it to forgetfulness and lack of compliance on the part of subjects faced with relatively unstructured eating patterns and significant amounts of food eaten away from home. In the present study, underreporting of foods eaten within our reference method undermines the process of its comparison with the other methods, and it is possible that the modest correlations we observed underestimate the true ability of FFQ1 to describe energy and macronutrient intake in this population.

Although we observed better agreement between the FCL and WDR in the assessment of energy and macronutrient intakes, energy intakes determined by the FCL were even lower than those from the WDR. If prospective recording techniques used by girls of this age result in underreporting of food intake, the better correspondence between the FCL and WDR could be misleading, merely indicating agreement between two similar types of dietary assessment method, which may both be flawed. We were disappointed in the performance of the FCL as it was far less onerous to complete than a WDR, subject compliance was greater, and it avoided the conceptualization and memory problems potentially associated with FFQs.

Since neither of the prospective methods used in the present study appeared to be good dietary assessment methods for use by girls of this age, can a FFQ be used instead to yield meaningful dietary information? In terms of compliance FFQ1 worked well. With the exception of one individual, it was completed successfully by all the girls in the study, estimates of intake were compatible with predicted energy needs (mean ratio of energy intake to predicted BMR $=1.67$ ), and it gave reproducible results when tested a month later. Overall there was reasonable agreement between FFQ1 and the reference method in terms of its description of the profile of diet and, with the exception of protein intakes, in its ability to estimate energy and macronutrient intake. FFQs have been developed for use by Norwegian and American adolescents $^{5,6}$, although compared with reference methods, modest correlations were observed. Since underreporting of food intake occurred in $20 \%$ of the weighed records against which the Norwegian FFQ was compared, Frost Andersen et al. ${ }^{5}$ also comment that the validity of their FFQ may have been underestimated.

Before using the FFQ in future we would need to address the problems encountered in its use in the present study. The Bland-Altman analyses ${ }^{16}$ indicated 
increasing difference in energy and macronutrient intakes between FFQ1 and the WDR as the level of intake rose. Although this may in part be due to incomplete recording of intake in the diaries it seems likely that there is overreporting of intake by a FFQ, and for FFQ1 this appeared to be particularly marked for soft drinks and fruit consumption. Whilst this raises the problem of defining the individual's absolute intakes of these foods, the relative ranking of girls may not be affected. A more serious problem is the poorer agreement between FFQ1 and the WDR in their estimation of cereal products, meat and fish, and snacks (confectionery, chocolate and savoury snacks) and sugar intakes. It is not possible to define why these particular food groups should be less well assessed, but given the relatively unstructured nature of the adolescent diet $^{2}$ the poorer agreement observed may result from too short a reference period when compared to an 'average' estimate of intake over a month by FFQ1, as well as to the apparent deficiencies within the WDR. In future, the inclusion of cross-check questions $^{20}$ to identify and adjust misreported intakes of fruit, soft drinks and meat and fish intakes should address some of these problems. Additionally we would continue to use a predefined cut-off to exclude records with implausibly high energy intakes.

Given the limitations discussed, the self-administered FFQ used by adolescent girls in the present study appears to yield reproducible responses which can be used to describe their broad dietary patterns. We suggest that the true ability of the FFQ used in the present study to assess diet in this population is greater than that shown by our data, owing to the nature of our study group and due to the quality of the dietary records used as the reference method. In general terms, the FFQ may represent a better dietary assessment tool for use with adolescents in the general population than prospective methods, and we would choose to use it again in the future. Although weighed records are the 'gold standard' when used by well-motivated individuals, other dietary assessment techniques are clearly needed for those individuals who are unwilling or unable to complete such records, and about whose diet little is known. The FFQ may represent one way to obtain meaningful dietary information from a wide variety of individuals including those who are unlikely to provide good prospective records.

\section{Acknowledgements}

We are very appreciative of the time spent on this study by all the girls who took part. We also thank the staff at the schools in Southampton who helped and facilitated the study. The study was funded by the Dunhill Trust and the Medical Research Council.

\section{References}

1 Godfrey K, Robinson S, Barker DJP, Osmond C, Cox V. Maternal nutrition in early and late pregnancy in relation to placental and fetal growth. BMJ 1996; 312: 410-14.

2 Livingstone MBE, Prentice AM, Coward WA, et al. Validation of estimates of energy intake by weighed dietary record and diet history in children and adolescents. Am. J. Clin. Nutr. 1992; 56: 29-35.

3 Bratteby LE, Sandhagen B, Fan H, Enghardt H, Samuelson G. Total energy expenditure and physical activity as assessed by the doubly labeled water method in Swedish adolescents in whom energy intake was underestimated by 7-d diet records. Am. J. Clin. Nutr. 1998; 67: 905-11.

4 Strain JJ, Robson PJ, Livingstone MBE, et al. Estimates of food and macronutrient intake in a random sample of Northern Ireland adolescents. Br. J. Nutr. 1994; 72: 343-52.

5 Frost Andersen L, Nes M, Lillegaard IT, Sandstad B, Bjorneboe G, Drevon CA. Evaluation of a quantitative food frequency questionnaire used in a group of Norwegian adolescents. Eur. J. Clin. Nutr. 1995; 49: 543-54.

6 Rockett HRH, Breitenbach M, Frazier AL, et al. Validation of a youth/adolescent food frequency questionnaire. Prev. Med. 1997; 26: 808-16.

7 Office of Population Censuses and Surveys. Standard Occupational Classification. London: HMSO, 1990.

8 Department of Health. Dietary Reference Values for Food Energy and Nutrients for the United Kingdom. London: HMSO, 1991.

9 Robinson S, Godfrey K, Cox V, Barker D. Evaluation of a food frequency questionnaire used to assess nutrient intakes in pregnant women. Eur. J. Clin. Nutr. 1996; 50: 302-8.

10 Crawley H. Food Portion Sizes. London: HMSO, 1988.

11 Davies J, Dickerson J. Nutrient Content of Food Portions. Cambridge: Royal Society of Chemistry, 1991.

12 Holland B, Unwin ID, Buss DH. Cereals and Cereal Products. Third Supplement to McCance and Widdowson's Composition of Foods. UK: Royal Society of Chemistry and Ministry of Agriculture, Fisheries and Food, 1988.

13 Holland B, Unwin ID, Buss DH. Milk, Milk Products and Eggs. Fourth Supplement to McCance and Widdowson's Composition of Foods. UK: Royal Society of Chemistry and Ministry of Agriculture, Fisheries and Food, 1989.

14 Holland B, Unwin ID, Buss DH. Vegetables, Herbs and Spices. Fifth Supplement to McCance and Widdowson's Composition of Foods. UK: Royal Society of Chemistry and Ministry of Agriculture, Fisheries and Food, 1991.

15 Holland B, Welch AA, Unwin ID, Buss DH, Paul AA, Southgate DAT. McCance and Widdowson's The Composition of Foods, 5th ed. UK: Royal Society of Chemistry and Ministry of Agriculture, Fisheries and Food, 1991.

16 Bland JM, Altman DG. Statistical methods for assessing agreement between two methods of clinical measurement. Lancet 1986; 1: 307-10.

17 Delcourt C, Cubeau J, Balkau B, Papoz L, CODIAB-INSERMZENECA Pharma Study Group. Limitations of the correlation coefficient in the validation of diet assessment methods. Epidemiology 1994; 5: 518-24.

18 Torun B, Davies PSW, Livingstone MBE, Paolisso M, Sackett R, Spurr GB. Energy requirements and dietary energy recommendations for children and adolescents 1 to 18 years old. Eur. J. Clin. Nutr. 1996; 50: S37-81.

19 Department of Health. The Diets of British Schoolchildren. London: HMSO, 1989.

20 Calvert C, Cade J, Barrett JH, Woodhouse A, UKWCS Steering Group. Using cross-check questions to address the problem of mis-reporting of specific food groups on food frequency questionnaires. Eur. J. Clin. Nutr. 1997; 51: $708-12$. 